\title{
Correction to: $P m 223899$, a new recessive powdery mildew resistance gene identified in Afghanistan landrace PI 223899
}

\author{
Genqiao $\mathrm{Li}^{1}$ - Brett F. Carver ${ }^{2}$. Christina Cowger ${ }^{3} \cdot$ Guihua Bai $^{4} \cdot$ Xiangyang Xu $^{1} \mathbb{C}$
}

Published online: 1 November 2018

○) Springer-Verlag GmbH Germany, part of Springer Nature 2018

\section{Correction to: Theoretical and Applied Genetics https://doi.org/10.1007/s00122-018-3199-y}

Unfortunately, the caption of Fig. 2 was incorrectly published in the original publication. The complete correct caption should read as follows.

Fig. 2 Graphical genotypes and phenotypes of critical $\mathrm{F}_{2}$ plants and corresponding $\mathrm{F}_{3}$ phenotypes. Pm223899 was mapped to an interval flanked by $P m 3 b-1$ and STARS333. Only one plant is shown for each genotype. R, S, HR, HS, and Seg represent resistant, susceptible, homozygous resistant, homozygous susceptible, and segregating, respectively
Also, under the "Discussion section", 3rd paragraph, the following sentence was incorrectly published and the complete correct sentence is given below.

There are 18 functional alleles at the Pm3 locus ( $P m 3 a$ Pm3r) (Yahiaoui et al. 2004, 2009; Bhullar et al. 2009, 2010), and one of them, $P m 3 a$, is widely used in the hard red winter wheat breeding programs in the Great Plains region (Li et al. 2016).

The original version is updated.

The original article can be found online at https://doi.org/10.1007/ s00122-018-3199-y.

Xiangyang Xu

xiangyang.xu@ars.usda.gov

1 Wheat, Peanut, and Other Field Crops Research Unit, USDA-ARS, Stillwater, OK 74075, USA

2 Plant and Soil Science Department, Oklahoma State University, Stillwater, OK 74078, USA

3 Plant Science Research Unit, USDA-ARS, Raleigh, NC 27695, USA

4 Hard Winter Wheat Genetics Research Unit, USDA-ARS, Manhattan, KS 66506, USA 